\title{
Cânceres da boca e faringe em crianças e adolescentes brasileiros: um estudo descritivo
}

\author{
Cancers of mouth and pharynx in Brazilian children and teenagers: a descriptive study
}

\author{
Shirley Suely S. V. Maciel'1, Wamberto Vieira Maciel², Willyanne Káthia S. de Vasconcelos ${ }^{3}$, Eduardo Sérgio D. Duarte Filho ${ }^{4}$, \\ Dellano Fernandes da S. Santos ${ }^{4}$, Gabriela Marques de Melo ${ }^{5}$
}

\section{RESUMO}

Objetivo: Descrever a mortalidade por cânceres da boca e faringe em crianças e adolescentes, dentre as neoplasias malignas registradas em brasileiros.

Métodos: Estudo retrospectivo de 167 óbitos de crianças (zero a 14 anos) e adolescentes (15 a 19 anos) registrados entre 1999 e 2005 no Brasil, e classificados nos códigos do Capítulo II (neoplasias-tumores) da CID-10, de acordo com a codificação de cânceres da boca e faringe (C00-C14). Os dados foram obtidos do Sistema de Informações sobre Mortalidade do Ministério da Saúde, disponibilizado em CD-ROM.

Resultados: A mortalidade proporcional por cânceres da boca e faringe na faixa etária em estudo representou $0,8 \%$ do total dos óbitos por todas as neoplasias e essa mortalidade aumentou com a idade $(0,26 \%$ para $<1$ ano e $1,52 \%$ para 15 a 19 anos). Entre zero a 14 anos ocorreram 69 óbitos (46 do sexo masculino e 23 do feminino) e, entre 15 e 19 anos, 98 óbitos (64 do sexo masculino e 34 do feminino), correspondendo a $0,5 \%(n=167)$ do total de óbitos por cânceres da boca e faringe registrados no Brasil.

Conclusões: A mortalidade foi maior em adolescentes do sexo masculino. O sítio anatômico que mais levou a óbito crianças e adolescentes foi a nasofaringe, sendo a orofaringe a topografia mais acometida da cavidade bucal.

Palavras-chave: neoplasias bucais; neoplasias faríngeas; mortalidade.

\section{ABSTRACT}

Objective: To describe the mortality in children and teenagers due to mouth and pharynx cancers among neoplasm cases registered in Brazil.

Methods: This is a retrospective study of 167 deaths of children (zero to 14 years old) and teenagers (15 to 19 years old) registered between 1999 and 2005 in Brazil, that were classified under the ICD-10 codes of mouth and pharynx cancers (C00-C14), in Chapter II. The data were obtained from the Information System about Mortality of the Ministry of Health, available in CD-ROM.

Results: The proportional mortality for mouth and pharynx cancers in the studied group represented $0.8 \%$ of the total deaths from all neoplasms. The mortality increased with age $(0.26 \%$ for $<1$ year old children and $1.52 \%$ for 15 to 19 years old teenagers). There were 69 deaths ( 46 males and 23 females) of children aged from zero to 14 years old and 98 deaths (64 males and 34 females) of teenagers from 15 to 19 years old, corresponding to $0.5 \%(n=167)$ of the total deaths caused by mouth and pharynx cancers recorded in Brazil.

Conclusions: Mortality was higher in male teenagers. The anatomic site that lead to more deaths of children and teenagers was the nasopharynx. Oropharynx was the most affected topography of the oral cavity.

Key-words: mouth neoplasms; pharyngeal neoplasms; mortality.
Instituição: Associação Caruaruense de Ensino Superior (Asces), Caruaru, PE, Brasil

'Doutora em Saúde Coletiva pela Universidade de Pernambuco (UPE); Professora da Faculdade do Vale do Ipojuca (Favip), Caruaru, PE, Brasil ${ }^{2}$ Mestre em Odontologia e em Morfologia pela UPE; Professor da Asces e da Favip, Caruaru, PE, Brasil

${ }^{3}$ Especialista em Saúde Pública pela Asces; Cirurgiã-Dentista da Secretaria de Saúde de Caruaru, Caruaru, PE, Brasil

${ }^{4}$ Graduado em Odontologia pela Asces; Professor da Associação Brasileira de Odontologia (ABO) - Regional Caruaru, Caruaru, PE, Brasil ${ }^{5}$ Graduado em Odontologia pela Asces; Cirurgião-Dentista da Secretaria de Saúde de Vitória de Santo Antão, Vitória de Santo Antão, PE, Brasil

${ }^{6}$ Graduanda do Curso de Odontologia da Asces, Caruaru, PE, Brasil
Endereço para correspondência:

Shirley Suely S. V. Maciel

Avenida Londres, 110 - Maria Gorete

CEP 55016-370 - Caruaru/PE

E-mail: shirleyverasmaciel@gmail.com

Conflitos de interesse: nada a declarar

Recebido em: 11/11/2009

Aprovado em: 1/6/2010 


\section{Introdução}

O câncer infantil compreende um grupo de doenças (tumores sólidos e doenças sistêmicas) que ocorre em qualquer parte do organismo, caracterizado pela divisão e proliferação desordenada de células que sofreram mutação em seu material genético, comprometendo tecidos e órgãos de indivíduos menores de $15 \operatorname{anos}^{(1,2)}$.

Estima-se uma incidência anual de cerca de 200 mil casos em todo o mundo ${ }^{(3)}$ e, no Brasil, de até 7 mil casos novos de câncer pediátrico por ano. Dados internacionais apontam que um em cada 100 mil adultos jovens será sobrevivente de um câncer na infância ${ }^{(2,4)}$. Nos países desenvolvidos, o câncer é a principal causa de óbito por doença em jovens até 15 anos, determinando $10 \%$ das mortes ${ }^{(5,6)}$, com tendência a aumento nos últimos anos ${ }^{(6)}$. Superado apenas pelos acidentes, o câncer ocupa a segunda posição ${ }^{(7)}$.

Os cânceres da boca e faringe configuram um problema de saúde pública, o que é verificado em estudos internacionais ${ }^{(8)}$ e do Brasil $^{(8,9)}$. As tendências de sobrevida para esses cânceres mostraram poucas alterações durante as últimas décadas no mundo ${ }^{(10)}$, principalmente pelo diagnóstico em estágio avançado da doença e pelas dificuldades de acesso aos serviços de diagnóstico precoce e tratamento, em algumas regiões ${ }^{(11)}$.

Dentre os cânceres da boca, mais de $90 \%$ são carcinomas de células escamosas (CEC), que poderiam ser facilmente identificados na cavidade bucal e bucofaríngea, tendo em vista o fácil acesso para o exame direto, sendo os mesmos responsáveis por $99 \%$ desses óbitos ${ }^{(12-14)}$. A etiologia desse câncer é multifatorial e, apesar de todo o avanço tecnológico obtido até o momento, seus agentes etiológicos ainda são uma incógnita ${ }^{(15)}$. Substâncias reconhecidamente carcinogênicas em pacientes mais idosos também podem estar relacionadas à etiologia do CEC em jovens ${ }^{(16)}$. Outros fatores devem ser investigados para explicar a doença em pacientes jovens: predisposição genética, infecção viral prévia, hábitos alimentares, estados de imunodeficiência, exposição ocupacional ao carcinógeno, condição socioeconômica e higiene oral ${ }^{(16)}$.

O presente estudo teve como objetivo descrever a mortalidade por cânceres da boca e faringe em 167 óbitos de crianças (zero a 14 anos) e adolescentes (15 e 19 anos) registrados no período de 1999 a 2005 no Brasil. Além disso, caracterizaram-se esses óbitos em relação às variáveis sexo, faixa etária, sítios anatômicos e as Unidades Federativas de residência, para que se direcionassem ações públicas e privadas por parte dos serviços de saúde, no sentido de minimizar o impacto social da afecção em estudo.

\section{Método}

Este é um estudo retrospectivo, que incluiu 167 óbitos de crianças e adolescentes (110 do sexo masculino e 57 do feminino) até 19 anos. Todos os óbitos foram registrados entre 1999 e 2005 no Brasil e divididos em dois grupos etários: o primeiro correspondeu às idades compreendidas entre zero e 14 anos (crianças ou óbitos infantis) e consistiu de 69 óbitos (46 do sexo masculino e 23 do feminino); o segundo, de 15 a 19 anos (adolescentes), constou de 98 óbitos (64 do sexo masculino e 34 do feminino), sendo classificados nos códigos do Capítulo II (neoplasias-tumores) da Classificação Internacional de Doenças na sua décima revisão (CID-10), de acordo com a codificação dos cânceres da boca e faringe (C00-C14).

O código usado para os cânceres de lábio foi o C00. O termo "câncer oral" pode ser encontrado na literatura e refere-se a todos os tipos de cânceres localizados na cavidade oral, inclusive a orofaringe, apresentando como sítios anatômicos a base da língua (C01), outras partes e partes não especificadas da língua (C02), gengiva (C03), assoalho da boca (C04), palato (C06), outras partes e partes não especificadas da boca (C06), glândulas parótidas (C07), glândulas salivares maiores e não especificadas (C08), amígdala (C09) e orofaringe $(\mathrm{C} 10)^{(17,18)}$. Os sítios anatômicos da faringe são nasofaringe (C11), seio piriforme (C12) e hipofaringe (C13); além disso, incluiu-se no presente estudo o código C14, no qual se enquadram os cânceres cujo sítio anatômico não foi identificado (outras localizações mal definidas do lábio, cavidade oral ou faringe).

Para análise da mortalidade, utilizou-se o banco de dados do Sistema de Informações sobre Mortalidade (SIM) do Ministério da Saúde, disponibilizado em CD-ROM. Optou-se por consultar a fonte de informação DATASUS por ser de fácil acesso, rápida e eficiente para obter os dados e informação em saúde, encontrando-se ao alcance de qualquer gestor, profissional ou pesquisador ${ }^{(19)}$. Porém, é uma base de dados ainda pouco explorada e analisada como ferramenta para ações de saúde pelo cirurgião-dentista.

Os óbitos por cânceres em crianças e adolescentes foram analisados por meio da mortalidade proporcional e dos coeficientes gerais e específicos de mortalidade, assim como as análises dos coeficientes de mortalidade por esses cânceres e sexo. A mortalidade proporcional para cada sexo e ano estudado foi calculada dividindo-se o número de óbitos por cânceres da boca e faringe pelo total de óbitos por cânceres ocorridos nessas faixas etárias, multiplicando-se por cem. 
Os coeficientes de mortalidade consistiram em uma razão em que, no numerador, estavam os óbitos por causa básica ocorridos em crianças e adolescentes residentes no Brasil e, no denominador, a população dessas mesmas faixas etárias, estimada para os respectivos anos do calendário. Os coeficientes de mortalidade foram expressos por meio do número de óbitos por essa causa básica por 100 mil habitantes dessa faixa etária. A população utilizada foi a do recenseamento de 2000 e demais contagens populacionais, obtida por meio do Instituto Brasileiro de Geografia e Estatística (IBGE).

A tabulação dos dados e o cálculo dos indicadores foram realizados por meio dos recursos do TabWin (tabulador de dados versão Windows) e do programa Microsoft ${ }^{\circledR}$ Office Excel 2003. Ressalte-se que, mesmo trabalhando-se com dados secundários publicados em CD-ROM pelo Ministério da Saúde, a pesquisa foi submetida à apreciação e aprovada pelo Comitê de Ética em Pesquisada Associação Caruaruense de Ensino Superior.

\section{Resultados}

Ao se comparar o número total de óbitos registrados em crianças e adolescentes em função dos cânceres $(n=21.441)$ com o número de óbitos por cânceres da boca e faringe nessa faixa etária ( $\mathrm{n}=167$ ), no período de 1999 a 2005, verificou-se uma mortalidade proporcional de $0,8 \%$, a qual aumentou com a idade de $0,26 \%$ (<1 ano) para 1,52\% (15 a 19 anos).

Os dados referentes ao total de óbitos por cânceres da boca e faringe, registrados no mesmo período, totalizaram 35.539, sendo que, destes, 167 ocorreram em crianças e adolescentes, representando 0,5\% do total. Embora seja uma porcentagem pequena, esse câncer representou, para a própria população em estudo, 15,0\% em 1999, atingindo 19,8\% em 2005.
A mortalidade por cânceres no Brasil e por cânceres da boca e faringe em crianças e adolescentes em números absolutos, a mortalidade proporcional e o coeficiente de mortalidade estão demonstrados na Tabela 1. Em números absolutos, os óbitos por cânceres da boca e faringe em crianças e adolescentes oscilaram no período estudado, passando de 25 em 1999 para 33, em 2005. Verificou-se uma oscilação dos seus valores em números absolutos, porcentagens e coeficientes, independentemente da faixa etária analisada. Todavia, esses valores foram maiores na faixa etária de 15 a 19 anos, excetuando-se em 1999, quando a doença levou a óbito $52,0 \%$ das crianças de zero a 14 anos acometidas por cânceres da boca e faringe. O coeficiente de mortalidade mais alto foi observado no ano de 2005 (0,09/100.000 habitantes).

Na Tabela 2, observa-se a distribuição dos óbitos por cânceres da boca e faringe segundo a faixa etária, o sexo e a raça das crianças e dos adolescentes, assim como o número absoluto e a porcentagem dos óbitos de acordo com o sítio anatômico do tumor. Do total de óbitos registrados no período em estudo, 110 ocorreram no sexo masculino, sendo a maioria ( $\mathrm{n}=54 ; 58,2 \%)$ em adolescentes. A maior parte dos óbitos ocorridos no sexo feminino $(\mathrm{n}=57)$ também foi observada em adolescentes $(n=34 ; 59,6 \%)$. A análise dos dados compreendeu ainda o estudo da razão entre sexos - masculino/feminino (M:F), cuja relação de óbitos de cânceres da boca e faringe vem se mantendo no mesmo padrão. Em 1999, a média era de três óbitos, sendo dois deles em homens para um em mulher (2:1). Já em 2005, a proporção permaneceu, sendo 1,9 homens para uma mulher (1,9:1).

Com relação à raça/cor da pele, a maior frequência de óbitos por câncer de boca e orofaringe foi notada em brancos $(n=79)$, seguidos pelos pardos ( $n=53)$, enquanto os negros e indígenas evidenciaram os menores números dentre o

Tabela 1 - Número, percentual e coeficiente de mortalidade por neoplasias malignas da boca e faringe, ano do óbito e faixa etária. Brasil, 1999-2005

\begin{tabular}{|c|c|c|c|c|c|c|c|c|c|}
\hline \multirow{3}{*}{ Ano } & \multicolumn{9}{|c|}{ Faixa etária (em anos) } \\
\hline & \multicolumn{3}{|c|}{$0-14$} & \multicolumn{3}{|c|}{$15-19$} & \multicolumn{3}{|c|}{$0-19$} \\
\hline & $\mathbf{n}$ & $\%$ & $\mathrm{CM}$ & $\mathbf{n}$ & $\%$ & CM & $\mathbf{n}$ & $\%$ & $\mathrm{CM}$ \\
\hline 1999 & 13 & 52,0 & 0,03 & 12 & 48,0 & 0,07 & 25 & 100,0 & 0,04 \\
\hline 2000 & 7 & 33,3 & 0,01 & 14 & 66,7 & 0,08 & 21 & 100,0 & 0,03 \\
\hline 2001 & 8 & 34,8 & 0,02 & 15 & 65,2 & 0,08 & 23 & 100,0 & 0,03 \\
\hline 2002 & 11 & 47,8 & 0,02 & 12 & 52,2 & 0,07 & 23 & 100,0 & 0,03 \\
\hline 2004 & 5 & 26,3 & 0,02 & 14 & 73,7 & 0,07 & 19 & 100,0 & 0,03 \\
\hline 2005 & 16 & 48,5 & 0,03 & 17 & 51,5 & 0,09 & 33 & 100,0 & 0,04 \\
\hline
\end{tabular}

Fonte: DATASUS/MS. CM: coeficiente de mortalidade para cada 100.000 habitantes. 
Tabela 2 - Distribuição dos óbitos por neoplasias malignas da boca e faringe segundo faixa etária, sexo, raça e sítio anatômico do tumor. Brasil, 1999-2005

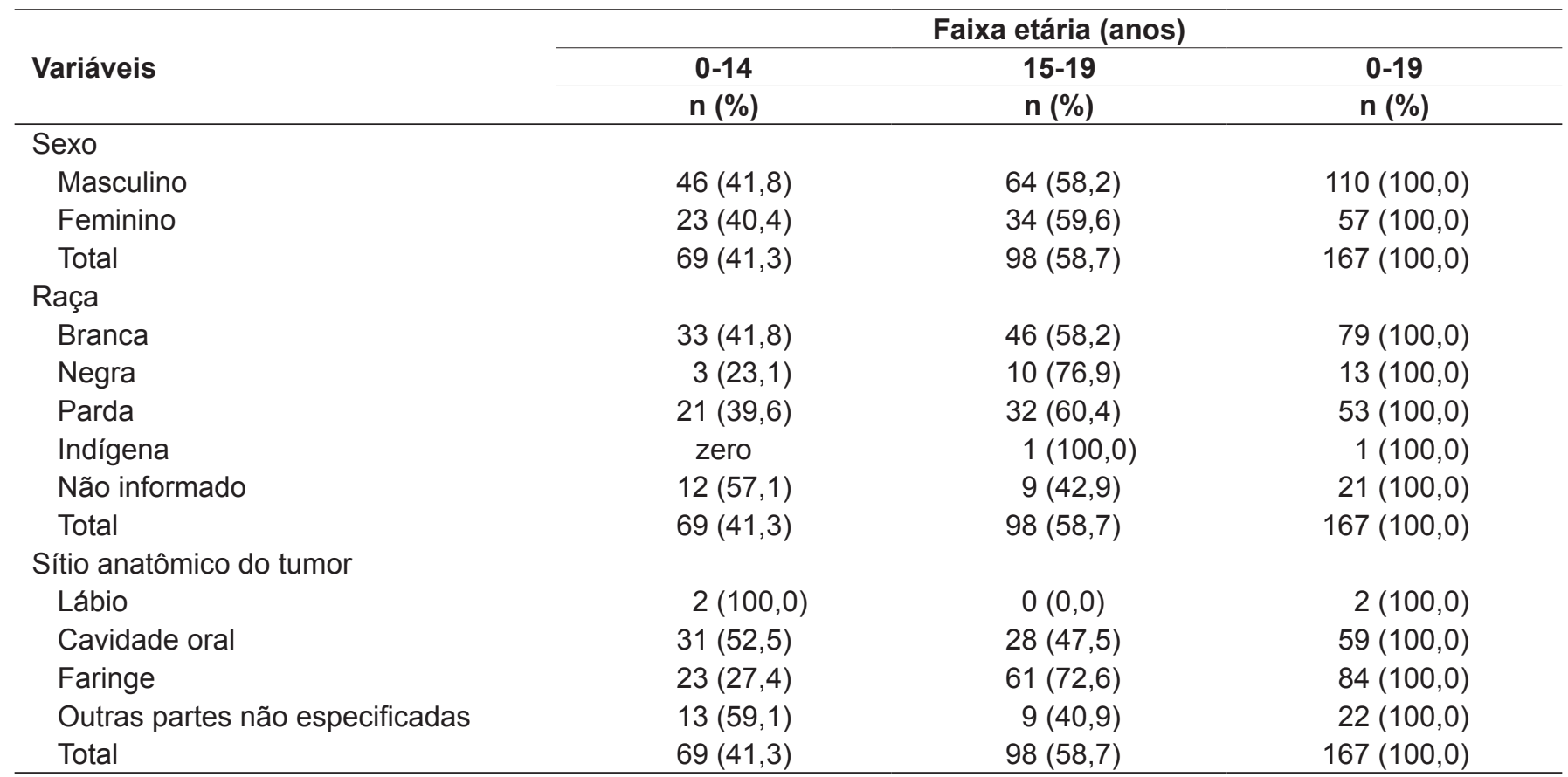

Fonte: DATASUS/MS.

total de óbitos (13 e 1, respectivamente). Todavia, adolescentes de cor negra foram vítimas fatais desses cânceres em frequência três vezes superior à observada para as crianças dessas categorias.

Os sítios anatômicos mais frequentemente atingidos foram faringe $(n=84)$ e cavidade oral $(n=59)$. Dentre os sítios anatômicos da faringe, o que mais levou crianças e adolescentes a óbito foi a nasofaringe $(49,1 \%)$ e, na cavidade bucal, a orofaringe $(9,6 \%)$, a língua $(5,4 \%)$, o palato $(4,8 \%)$ e as glândulas salivares $(3,6 \%)$.

Entretanto, ao se analisarem os óbitos por faixa etária, verificou-se que o sítio anatômico mais atingido em crianças pelos cânceres foi a cavidade oral $(\mathrm{n}=31 ; 52,5 \%)$ e, em adolescentes, a faringe $(n=61 ; 72,6 \%)$. Chama a atenção o número de sítios anatômicos que deixaram de ser diagnosticados $(n=22)$, principalmente em crianças $(n=13 ; 59,1 \%)$ e nos Estados da Bahia $(n=4)$, Minas Gerais $(n=3)$ e Paraná $(n=3)$, representando 45,4\% dessas informações.

Quanto à distribuição dos óbitos segundo os estados, houve predomínio dos óbitos em São Paulo ( $\mathrm{n}=31$ ), seguido por Minas Geras $(n=17)$, Paraná $(n=15)$ e Rio de Janeiro $(\mathrm{n}=14)$. Note-se que apenas $28,7 \%(\mathrm{n}=48)$ dos óbitos foram registrados em capitais brasileiras, sendo São Paulo $(n=8)$, Rio de Janeiro ( $\mathrm{n}=8)$ e Brasília $(\mathrm{n}=6)$ as capitais com o maior número de registros.

\section{Discussão}

Neste estudo, algumas questões merecem reflexão, por exemplo, o fato de que o uso de dados secundários não permite ao pesquisador controlar possíveis erros decorrentes de digitação e registro, além de possíveis sub-registros. Alguns autores apontam que o maior número de sub-registros por câncer bucal é encontrado nas capitais brasileiras menos desenvolvidas ${ }^{(20)}$. Outra questão refere-se à necessidade de uma melhor investigação dos diagnósticos desses cânceres ocorridos em crianças menores de um ano, visto que todos foram registrados em crianças residentes no interior dos Estados.

Sugere-se, assim, que os resultados sejam analisados com cautela, devido às diferenças existentes entre a disponibilidade de serviços de saúde em cada região e também à capacitação das pessoas que atestam a declaração de óbito, apesar de o sistema de informação ser o mesmo. Todavia, apesar dessas ressalvas, acredita-se que, por se tratar de dados nacionais oficiais e da obrigatoriedade do preenchimento das declarações de óbitos em todos os serviços de saúde, seus resultados permitiram o alcance dos objetivos propostos.

Ao encontrar uma baixa porcentagem de óbitos por cânceres da boca e faringe em crianças (zero a 14 anos) e adolescentes (15 a 19 anos), este estudo retrospectivo reforça a informação de que tais doenças acometem indivíduos mais 
velhos e que a mortalidade por esses cânceres aumenta com a idade. Ao comparar essas duas faixas etárias, observou-se que o maior número de óbitos foi registrado em adolescentes de 15 aos 19 anos, em relação às crianças de zero a 14 anos, em ambos os sexos, o que corrobora outros estudos ${ }^{(18,21)}$ que comprovaram a existência de uma associação inversamente proporcional entre a mortalidade infantil por câncer da boca e a idade.

Outro fato que pode justificar os achados do presente estudo é que o CEC, câncer mais comum da boca e orofaringe ${ }^{(12-14,22)}$, afeta principalmente adultos com mais de 50 anos, atingindo valores máximos aos 80 anos $^{(11)}$, sendo rara sua ocorrência em indivíduos com idade inferior a 40 anos (1 a $6 \%$ dos casos) ${ }^{(16,23-25)}$. O baixo percentual de mortalidade em crianças menores de um ano encontrado na presente pesquisa está de acordo com informações citadas na literatura ${ }^{(26)}$ que apontam os carcinomas como o principal grupo de câncer incidente na boca ${ }^{(12-14,22)}$, sendo mais frequentes entre dez e 14 anos. Dois estudos ajudam a compreender esses achados ao mostrarem que a maioria das crianças atingidas pelos cânceres infantis tem de zero a $4 \operatorname{anos}^{(26)}$ e que, dentre 35,9\% das crianças acometidas nessa faixa etária, somente 3,4\% possuem até um ano ${ }^{(27)}$.

No presente estudo, verificou-se que os coeficientes de mortalidade vêm sofrendo pequenas oscilações, observandose o mais alto coeficiente no último ano da pesquisa (2005). Contudo, a literatura aponta que, a cada ano, a incidência de câncer ${ }^{(5)}$ está aumentando e, no Brasil, nas idades de zero a 18 anos, há 12 a 16 novos casos de câncer por ano para cada 100 mil indivíduos, chegando a ser a terceira causa de morte na população abaixo de 14 anos $^{(28)}$.

Alguns autores consideram que a lesão pré-cancerígena em jovens é particularmente agressiva, apresentando pior prognóstico do que em pacientes mais idosos. Estudos realizados $^{(24,29)}$ concluíram que jovens tendem a apresentar maior taxa de recorrência locorregional e menor sobrevida, enquanto outros ${ }^{(30,31)}$ descreveram prognóstico similar nos dois grupos etários.

É notória a significativa agressividade do câncer em crianças e adolescentes. No adulto, em muitas situações, o surgimento do câncer está associado aos fatores ambientais, por exemplo, fumo e câncer de pulmão, enquanto nas malignidades da infância não se observa claramente tal associação ${ }^{(32)}$. Inúmeras pesquisas indicam que os mecanismos causais e a etiologia do câncer infantil são multifatoriais, envolvendo exposições a agentes físicos, químicos e biológicos, efeitos risco-moduladores de defeitos genéticos, ação de alguns alimentos, além do acaso. Casos de predisposição genética hereditária correspondem a menos de $3 \%$ dos cânceres da criança e do adolescente; fatores ocupacionais dos pais antes ou durante a gestação também parecem intervir na ocorrência de tumores no período neonatal e em lactentes ${ }^{(7,26)}$. Grupos populacionais de baixa renda tendem a apresentar precárias condições de saúde bucal e carências nutricionais, achados comuns entre os casos de cânceres da boca e faringe ${ }^{(33-35)}$.

A análise por sexo evidenciou que, independentemente da faixa etária em estudo, esses cânceres levaram a óbito mais pessoas do sexo masculino, concordando com outros estudos $^{(15)}$, o que resultou em alguns questionamentos, como, por exemplo, se há uma maior suscetibilidade do sexo masculino a essas neoplasias, tendo em vista que não se pode atribuir a estes as mesmas justificativas utilizadas às pessoas com idades mais avançadas. Em se tratando de crianças e adolescentes, não se pode dizer que essas demoraram a procurar os serviços de saúde ou que tiveram seus tratamentos negligenciados, bem como não se pode afirmar que homens fumam ou bebem mais do que mulheres nessa faixa etária. Entretanto, ao estudar a incidência dos cânceres da boca e faringe em Recife (PE), entre 1995 e 2001, Castro et al ${ }^{(11)}$ chamaram atenção para o elevado percentual desses cânceres no sexo feminino, em crianças e adolescentes de zero a 19 anos $(12,0 \%)$ e adultos jovens de 20 a 39 anos $(8,6 \%)$. Acredita-se que novas pesquisas possam contribuir para o conhecimento de uma possível maior suscetibilidade desses cânceres no sexo feminino, na faixa etária em estudo, tanto em relação à doença quanto ao óbito.

Parece não haver associação entre a cor da pele e a mortalidade por câncer infantil ou a sua incidência; porém, percebe-se que os mais atingidos são crianças e adolescentes da cor branca. Estudo que analisou os prontuários de 495 pacientes atendidos em uma unidade de oncologia pediátrica em Salvador, Bahia ${ }^{(27)}$, mostrou que $51 \%$ dos pacientes eram pardos e $21 \%$, brancos.

Encontrou-se, no presente estudo, que um dos sítios de maior ocorrência do câncer bucal em jovens foi a língua, similar ao verificado em pacientes de faixa etária mais elevada ${ }^{(11,23)}$, parecendo não haver distinção entre esse sítio anatômico e as faixas etárias, conforme já destacado na literatura ${ }^{(24)}$. Em relação à comparação entre faringe e cavidade oral, a maioria dos óbitos em crianças e adolescentes adveio do câncer da nasofaringe, em concordância com a maior incidência desse carcinoma descrita na literatura $^{(36)}$. Todavia, outros autores ${ }^{(11)}$ 
verificaram um maior número de casos novos na cavidade bucal (72,3\%), comparativamente à faringe. Tal discordância pode ser explicada por uma eventual maior agressividade do tumor maligno nesse locus - necessitando-se, pois, de pesquisas que comprovem essa hipótese - ou também pode ser atribuída às divergências regionais existentes no país. Conforme relatado na literatura ${ }^{(7)}$, este estudo está de acordo com a maior prevalência de óbitos por câncer em jovens nas regiões mais industrializadas do país, mas não necessariamente nas capitais.

Cabe ressaltar o fato de que, apesar de a utilização de dados secundários apresentar algumas vantagens, notificações incompletas ou campos deixados em branco dificultam o conhecimento do verdadeiro panorama da mortalidade por essa doença, visto que foi grande o número de dados ignorados sobre a identificação do sítio anatômico do tumor. Isso

\section{Referências bibliográficas}

1. Teixeira RA, Bruniera P, Cusato MP, Borsato ML. Câncer infantil. In: Baracat FF, Fernandes-Junior HJ, Silva MJ. Cancerologia atual: um enfoque multidisciplinar. São Paulo: Roca; 2000. p. 426-51.

2. Haagedoorn EM, Oldhoff J, Bender W, Clarke WD, Sleijfer DT. Oncologia básica para profissionais de saúde. São Paulo: Associação Paulista de Medicina; 2000.

3. Vargas PL. Cáncer en pediatría: aspectos generales. Rev Chil Pediatr 2000;71:283-95.

4. Ward JD. Pediatric cancer survivors: assessment of late effects. Nurse Pract 2000;25:18.

5. Instituto Nacional de Câncer [homepage on the Internet]. Peculiaridades do câncer infantil [cited 2009 Jan 11]. Available from: http://www.inca.gov.br/ conteudo_view.asp?id=343

6. Seguin RC. O câncer na região da cabeça e pescoço e o trabalho corporal sobre o enfoque da bioenergética [monografia na Internet] Americana: Instituto Ligare; 2003 [citado em Junho 20, 2009]. Available from: http://ligare.psc.br/ lista_teses.php?teses_id=5

7. Aguiar SS. Epidemiologia do câncer pediátrico. In: Centro Infantil Boldrini, editor. Quando suspeitar do câncer na criança e no adolescente. Campinas: Centro Infantil Boldrini. [cited 2009 Jan 11] Available from: http://www.manualssearch-engine.com/download/respostas-boldrini-3.html

8. Franceschi D, Gupta R, Spiro RH, Shah JP. Improved survival in the treatment of squamous carcinoma of the oral tongue. Am J Surg 1993;166:360-5.

9. Dedivitis RA, França CM, Mafra AC, Guimarães FT, Guimarães AV. Características clínico-epidemiológicas no carcinoma espinocelular de boca e orofaringe. Rev Bras Otorrinolaringol 2004;70:35-40.

10. Blot WJ, McLaughlin JK, Devesa SS, Fraumeni Jr JF. Cancers of the oral cavity and pharynx. In: Schottenfeld D, Fraumeni Jr JF, editors. Cancer epidemiology and prevention. New York: Oxford University Press; 1996. p. 666-80.

11. Castro LS, Barros EC, Maciel SS, Gondim LA, Maciel WV, Oliveira Júnior FJ. The incidence of the oral and oropharyngeal cancers in Recife-PE, at period of 1995 to 2001. Revista da AMRIGS 2008;52:158-63.

12. Barbosa JF, Fonseca EP. Câncer bucal: diagnóstico, tratamento e reabilitação. Brasília: Ministério da Saúde; 1972.

13. Cantazaro Guimarães SA. Patologia básica da cavidade bucal. Rio de Janeiro: Guanabara Koogan; 1982. sugere o despreparo dos médicos em atestar corretamente o óbito, constituindo-se em barreira importante para o tratamento dessas neoplasias malignas, já que existem diferenças na forma de tratamento das mesmas.

Apesar de não ter sido objeto do estudo conhecer os fatores de risco associados aos cânceres da boca e faringe, a descrição da mortalidade de crianças e adolescentes entre zero a 19 anos indica a necessidade de novos estudos para fornecer conhecimento mais acurado das características dessa população e dos riscos aos quais ela está exposta. É também preciso apontar para a necessidade de melhoria da qualidade dos dados em algumas unidades federativas brasileiras, para que o Sistema de Informações sobre Mortalidade torne-se uma ferramenta útil para os pediatras e odontopediatras repensarem sua prática, em função da quantidade de óbitos ocorrida, principalmente em adolescentes.

14. Waldron CA. Oral epithelial tumors. In: Gorlin RJ, Goldman HM. Thoma's Oral Patolog. Saint Louis: Mosby; 1970. p. 801-60.

15. Neville BW, Damm DD, Allen CM, Bouquot JE. Patologia oral e maxilofacial. $2^{a}$ ed. Rio de Janeiro: Guanabara Koogan; 2004.

16. Llewellyn CD, Johnson NW, Warnakulasuriya KA. Risk factors for squamous cell carcinoma of the oral cavity in young people - a comprehensive literature review. Oral Oncol 2001;37:401-18.

17. Instituto Nacional de Câncer. TNM: classificação dos tumores malignos. $6^{a}$ ed. Rio de Janeiro: Instituto Nacional de Câncer; 2004.

18. Maciel SS. Tendências da mortalidade por câncer bucal no Brasil: análise do período de 1991 a 2002 [tese de doutorado]. Recife (PE): UFPE; 2004.

19. da Silva EC, da Costa Junior ML. Mental and behavioral disturbances in the hospital information system: perspectives for nursing. Rev Esc Enferm USP 2006;40:196-202.

20. Borges DML, Sena MF, Ferreira MAF, Roncalli AG. Mortalidade por câncer de boca e condição sócio-econômica no Brasil. Cad Saude Pública 2009; 25:321-7.

21. Marques $F$ [homepage on the Internet]. Regiões mais ricas têm maior mortalidade por câncer de boca. Diário da Saúde [cited 2009 Jul 12]. Available from: http://diariodasaude.com.br/news.php?article=regioes-mais-ricas-temmaior-mortalidade-por-cancer-de-boca

22. Tincani AJ, Brandalise N, Andreollo NA, Lopes LR, Montes CG, Altemani A et al. A importância da endoscopia digestiva alta com solução de lugol no diagnóstico de câncer superficial e displasia em esôfago de doentes com neoplasias de cabeça e pescoço. Arq Gastroenterol 2000;37:107-13.

23. Burzynski NJ, Flynn MB, Faller NM, Ragsdale TL. Squamous cell carcinoma of the upper aerodigestive tract in patients 40 years of age and younger. Oral Surg Oral Med Oral Pathol 1992;74:404-8.

24. Torossian JM, Beziat JL, Philip T, Bejui FT. Squamous cell carcinoma of the tongue in a 13-year-old boy. J Oral Maxillofac Surg 2000;58:1407-10.

25. Hirota SK, Migliari DA, Sugaya NN. Oral squamous cell carcinoma in a young patient: case report and literature review. An Bras Dermatol 2006;81:251-4.

26. Mirra AP, Latorre MR, Veneziano DB. Incidência, mortalidade e sobrevida do câncer da infância no Município de São Paulo. São Paulo: Registro de Câncer de São Paulo; 2004. 
27. Diniz AB, Regis CA, Brito NP, Conceição LS, Moreira LM. Perfil epidemiológico do câncer infantil em população atendida por uma unidade de oncologia pediátrica em Salvador-Bahia. Rev Ciênc Méd Biol 2005;4:131-9.

28. Mendonça N. Porque o câncer deve ser considerado como doença "própria da infância". Pediatria 2000;76:261-2.

28. Sarkaria JN, Harari PM. Oral tongue cancer in young adults less than 40 years of age: rationale for aggressive therapy. Head Neck 1994;16:107-11.

30. Friedlander PL, Schantz SP, Shaha AR, Yu G, Shah JP. Squamous cell carcinoma of the tongue in young patients: a matched-pair analysis. Head Neck 1998;20:363-8.

31. Schantz SP, Byers RM, Goepfert H, Shallenberger RC, Beddingfield N. The implication of tobacco use in the young adult with head and neck cancer. Cancer 1988;62:1374-80.
32. Hospital do Câncer de Mato Grosso [homepage on the Internet]. Câncer infantil: particularidades [cited 2008 May 20]. Available from: http://hcancer.com.br

33. La Vecchia C, Tavani A, Franceschi S, Levi F, Corrao G, Negri E. Epidemiology and prevention of oral cancer. Oral Oncol 1997;33:302-12.

34. Antunes JL, Biazevic MG, de Araujo ME, Tomita NE, Chinellato LE, Narvai PC Trends and spatial distribution of oral cancer mortality in São Paulo, Brazil, 1980-1998. Oral Oncol 2001;37:345-50.

35. Greenwood M, Thomson PJ, Lowry RJ, Steen IN. Oral cancer: materia deprivation, unemployment and risk factor behaviour - an initial study. Int J Oral Maxillofac Surg 2003;32:74-7.

36. Liga Paranaense de Combate ao Câncer. Câncer na infância e na adolescência. Curitiba: LPCC; 2004 